\title{
Primary and secondary prevention of human papillomavirus-associated cancer: it's not all about women
}

\author{
Simona Venturoli, ${ }^{1}$ Vittorio Sambri ${ }^{2,3}$ \\ ${ }^{1}$ Microbiology and Virology Unit, Bologna University Hospital, Sant'Orsola-Malpighi Policlinic, Bologna; ${ }^{2}$ Microbiology \\ Unit, Romagna Local Health Unit, Pievesestina; ${ }^{3}$ DIMES, University of Bologna, Italy
}

To date, we have two important weapons in the prevention of uterine cervical cancer: vaccine program and organized screening. The most important task for the prevention of cervical cancer is to accelerate an integrated approach of vaccination and screening. Furthermore, High Risk Human Papillomavirus (HR-HPV) persistent infections are linked to other cancers in addition to cervical cancer: these are including localization in the vulva, vagina, penis, anal canal and head and neck. In the developed countries, the number of HPV-related cancers in men, including penile, oral, and anal cancer, is like that of cervical cancer in women. Additional morbidity due to HPV in men results from development of genital warts and HPV infection in men leads to substantial morbidity and mortality in women (9). Therefore, it is clear that HPV infection in men is a serious clinical issue.

Effective prevention strategies (vaccine and screening) for cervical cancer may be applied, at least in theory, to the prevention of other HPV associated tumors that also affect males.

Nowadays vaccination of girls against the HPV is implemented in most developed western countries. In Italy vaccination of young adolescent girls with prophylactic HPV vaccines (bivalent or quadrivalent) has been rolled out as a Public Health Programme from 2008. Based on clinical trials $(5,11)$, the US Food and Drug Administration (FDA) approved the extension of the indications for

Correspondence: Simona Venturoli, U.O. Microbiologia e Virologia, Policlinico S. Orsola Malpighi, via G. Massarenti 9, 40138 Bologna, Italy.

Tel.: +39.051.2144512 - Fax: +39.051.307397

E-mail: simona.venturoli@unibo.it

Key words: Papillomavirus; HPV-related cancer; vaccine; screening; male.

Contributions: SV wrote the manuscript, VS revised the paper.

Conflict of interest: the authors declare no potential conflict of interest.

Received for publication: 27 June 2017.

Accepted for publication: 27 June 2017.

(C) Copyright S. Venturoli and V. Sambri, 2017

Licensee PAGEPress, Italy

Microbiologia Medica 2017; 32:6868

doi:10.4081/mm.2017.6868

This article is distributed under the terms of the Creative Commons Attribution Noncommercial License (by-nc 4.0) which permits any noncommercial use, distribution, and reproduction in any medium, provided the original author(s) and source are credited. the quadrivalent vaccine to males up to the age of 26 years for the prevention of anogenital warts (FDA October 2009) and for the prevention of anal cancer and anal intraepithelial lesions in males and females aged between 9 and 26 years (FDA December 2010). Although HPV vaccination effectively protects against external genital lesions and anal intraepithelial neoplasia in males, only a few countries (USA, Australia, Austria, Canada, Germany limited to the Saxony region) have recommended universal vaccination of boys. In other countries, a cautious approach has been due to uncertainties around the population level impact and cost effectiveness of vaccination of boys. Some countries have chosen to introduce the vaccine for the prevention of anal cancer only in populations with increased risk like men who have sex with men (MSM) (2).

Few studies to date have been conducted to evaluate the impact of the HPV vaccine on oral HPV infection or oropharyngeal cancer (OPC) $(4,7)$. On the other hand, some studies have used predictive modelling to understand the potential benefit of HPV vaccination within the context of OPC (OPCs now account for $78 \%$ of newly diagnosed HPV-related tumors in men) (6).

Based on the new and important scientific evidence, the Italian National Plan for Vaccine Prevention (2017-2019) recently published targets the immunization of adolescents of both sexes, for maximum protection against all directly related HPV-related pathologies and to stop the circulation of the virus.

At the same time as the implementation of the vaccine program, several countries have decided to, or are considering switching from cytology based screening to HPV-based screening (11). In Italy, the introduction of HPV-based screening in routine activity is starting at different times in different regions and in the 2021 the cohorts of women who were offered HPV vaccination (twelve-year-old cohorts) will be reaching the age for screening and this will lead to changes in the currently scheduled screening program.

With the existence of well-established cervical cancer screening programmes and linked cytology, cancer and HPV vaccine registries, it is possible to directly monitor early end points, namely reduction in screen-detected cervical abnormalities, following implementation of the HPV vaccination programme in females. In contrast to cervical screening programmes, population-wide screening for HPV infection or related disease in males is not recommended. However, direct monitoring of early end points of male vaccination is challenging given the absence of any equivalent screening initiatives and therefore the evaluation of HPV vaccine effectiveness in males will require dedicated surveillance strategies (3).

Regarding secondary prevention, currently no evidences are available supporting screening for vulvar, vaginal or penile cancer. The possibility of a screening strategy for the prevention of HPVrelated tumors in males requires, however, some considerations. While the population prevalence of penile HPV-related cancers is 
too low for population-based screening, anal cancer incidence is higher in HIV-positive men who have sex with men (MSM), warranting consideration of early detection approaches (10). For anal cancer screening HPV testing and cytology may be most effective for their negative predictive value when both are negative by indicating an individual who would not need high resolution anoscopy, because their risk of having high grade anal intraepithelial neoplasia (HGAIN) is extremely low. For HIV-negative MSM, HPV testing added to cytology testing in a similar manner to its use in the cervix may be helpful in identifying men with the greatest risk of HGAIN. Larger clinical trials are necessary to determine the optimum method of anal screening (1) and to ensure that appropriate evidence is available supporting population level effectiveness and cost-effectiveness.

In the head and neck region no specific HPV-associated precancerous lesions could be identified to date hampering early detection of HPV-induced neoplasia and then a screening for oropharyngeal cancer does not seem feasible, however the accurate detection of HPV-induced oropharyngeal cancer plays an important role to select patients that may benefit from a de-escalated treatment schemes (11).

Vaccine and screening programs are complex entities and establishing or changing programs requires considerable investment to ensure that appropriate evidence is available supporting population level effectiveness and cost-effectiveness. Based on the evidence available, the importance of introducing HPV vaccination of boys is obvious (8), while screening for penile cancer is not justified based on low incidence of disease and cost effectiveness. Anal cancer screening is to be considered but will provide more indepth studies to define target populations (HIV + males and females, MSM, etc.).

\section{References}

1. Berry JM, Palefsky JM, Jay N, et al. Performance characteristics of anal cytology and human papillomavirus testing in patients with high-resolution anoscopy-guided biopsy of highgrade anal intraepithelial neoplasia. Dis Colon Rectum 2009;52:239-47.

2. Bogaards JA, Wallinga J, Brakenhoff RH, et al. Direct benefit of vaccinating boys along with girls against oncogenic human papillomavirus: Bayesian evidence synthesis. BMJ 2015;350: 2016.

3. Garland SM, Molesworth EG, Machalek DA, et al. How to best measure the effectiveness of male human papillomavirus vaccine programmes? Clin Microbiol Infect 2015; 21:834-41.

4. Gillison ML. Human papillomavirus-related diseases: oropharynx cancers and potential implications for adolescent HPV vaccination. J Adolesc Health 2008;43:S52-60.

5. Giuliano AR, Palefsky JM, Goldstone S, et al. Efficacy of quadrivalent HPV vaccine against HPV infection and disease in males. N Engl J Med 2011;364:401-11.

6. Graham DM, Isaranuwatchai W, Habbous S, et al. A costeffectiveness analysis of human papillomavirus vaccination of boys for the prevention of oropharyngeal cancer. Cancer 2015;121:1785-92.

7. Guo T, Eisele DW, Fakhry C. The potential impact of prophylactic human papillomavirus vaccination on oropharyngeal cancer. Cancer 2016;122:2313-23.

8. Lenzi A, Mirone V, Gentile V, et al Rome Consensus Conference - statement; human papilloma virus diseases in males. BMC Public Health 2013;13:117.

9. Palefsky JM. Human papillomavirus-related disease in men: not just a women's issue. J Adolesc Health 2010;46:S12-9.

10. Prigge ES, von Knebel Doeberitz M, Reuschenbach M. Clinical relevance and implications of HPV-induced neoplasia in different anatomical locations. Mutat Res 2017; 772:51-66.

11. Wentzensen N, Arbyn M, Berkhof J, et al. Eurogin 2016 roadmap: how HPV knowledge is changing screening practice. Int J Cancer 2017;140:2192-200. 